\title{
Information measures for record ranked set samples
}

\author{
Maryam Eskandarzadeh ${ }^{1}$, Saeid Tahmasebi ${ }^{* 2}$ and Mahmoud Afshari ${ }^{3}$ \\ 1,2,3 Department of Statistics, Persian Gulf University, Bushehr, Iran
}

\begin{abstract}
Salehi and Ahmadi (2014) introduced a new sampling scheme for generating record-breaking data called record ranked set sampling. In this paper, we consider the uncertainty and information content of record ranked set samples (RRSS) in terms of Shannon entropy, Rényi and Kullback-Leibler $(K L)$ information measures. We show that the difference between the Shannon entropy of RRSS and the simple random samples (SRS) is depends on the parent distribution F. We also compare the information content of RRSS with a SRS data in the uniform, exponential, Weibull, Pareto, and gamma distributions. We obtain similar results for RRSS under the Rényi information. Finally, we show that the KL information between the distribution of SRS and distribution of RRSS is distribution-free and increases as the sample size increases.
\end{abstract}

Keywords: Kullback - Leibler information, Record ranked set sampling design, Rényi Information, Shannon entropy. 


\section{Introduction}

Suppose that $\left\{X_{n}, n \geq 1\right\}$ is a sequence of independent and identically distributed random variables with the cumulative distribution function (cdf) $F$. Also, let

$$
Y_{n}=\max (\min )\left\{X_{j} \mid 1 \leq j \leq n\right\} .
$$

We say $X_{j}$ is an upper (lower) record value of $\left\{X_{n}, n \geq\right.$ $1\}$, if $Y_{j}>(<) Y_{j-1}, j>1$. Let the 1th upper and lower record be taken as $L_{1}=U_{1} \equiv X_{1}$, and denote the nth ordinary upper and lower record by $U_{n}$ and $L_{n}$, respectively (for $n \geq 1$ ). These type of data arise in a wide variety of practical situations such as industrial stress testing (Samaniego and Whitaker, 1986 ), meteorology (Benestad, 2003), biology (Krug and Jain, 2005), sports (Kuper and Sterken, 2003), and stock market analysis (Bradlow and Park, 2007 ). Interested readers refer to the books by Ahsanullah (1995) and Arnold et al.(1998). Ranked set sampling was first proposed by McIntyre (1952) for estimating the mean pasture yields. McIntyre (1952) indicates that ranked set sampling is a more efficient sampling method than simple random sampling method for estimating the population mean. In the ranked set sampling technique, the sample selection procedure is composed of two stages. At the first stage of sample selection, $n$ simple random samples of size $n$ are drawn from an infinite population and each sample is called a set. Then, each of observations are ranked from the smallest to the largest according to variable of interest, say $X$, in each set. Ranking of the units is done with a low-level measurement such as using previous experiences, visual measurement or using a concomitant variable. At the second stage, the first observation unit from the first set, the second observation unit from the second set and going on like this $n$ th-observation unit from the $n$ thset are taken and measured according to the variable $X$ with a high level of measurement satisfying the desired sensitivity. The obtained sample is called an ranked set sample(RSS). In fact, the proposed scheme is motivated based on the ordinary ranked set sampling which was introduced by McIntyre (1952). This sampling, in turn, yields more efficient estimators of many population parameters of interest (such as mean, median, variance, quantiles) than a simple random sample (SRS) of the same size does(see Chen et al., 2004). The one-cycle RSS of size $m$ can be demonstrated as below:

$$
\begin{array}{ccccc}
1: & \mathbf{X}_{(\mathbf{1 : m}) \mathbf{1}} & X_{(2: m) 1} & \cdots & X_{(m: m) 1} \rightarrow X_{1,1}=X_{(1: m) 1} \\
2: & X_{(1: m) 2} & \frac{\mathbf{X}_{(2: \mathbf{m}) 2}}{\cdots} & X_{(m: m) 2} \rightarrow X_{2,2}=X_{(2: n) 2} \\
\vdots & \vdots & \vdots & \ddots & \vdots \\
n: & X_{(1: m) m} & X_{(2: m) m} & \cdots & \mathbf{X}_{(\mathbf{m}: \mathbf{m}) \mathbf{m}} \rightarrow X_{m, m}=X_{(m: m) m}
\end{array}
$$

where $X_{(i: m) j}$ denotes the ith order statistic from the $j$ th simple random sample of size $m$. The vector of observations $\mathbf{X}_{R S S}=\left(X_{1,1}, \ldots, X_{m, m}\right)$ is a one-cycle RSS of size $m$; note that $X_{i, i}$ 's are not necessarily ordered. Recently, Salehi and Ahmadi(2014) introduced a new sampling scheme for generating record data. Suppose we have $m$ independent sequences of continuous random variables. The ith sequence sampling is terminated when the ith record is observed. The only observations available for analysis are the last record value in each sequences. Salehi and Ahmadi(2014) called this design the record ranked set sampling. In fact, the proposed scheme is based on general RSS which is a sampling procedure that can be viewed as a generalization of the SRS. Let us denote the last record for the $i$ th sequence by $R_{i, i}$, if $\mathbf{R}=\left(R_{1,1}, R_{2,2}, \ldots, R_{m, m}\right)$ is a RRSS of size $m$, then the following procedure is used for representing this design

$$
\begin{aligned}
& 1: \mathbf{R}_{(\mathbf{1}) \mathbf{1}} \quad \rightarrow \quad R_{1,1}=R_{(1) 1} \\
& 2: \frac{R_{(1) 2}}{R_{(2) 2}} \quad \rightarrow \quad R_{2,2}=R_{(2) 2} \\
& m: \quad \begin{array}{cccc}
R_{(1) m} & R_{(2) m} & \cdots & \underline{\mathbf{R}_{(\mathbf{m}) \mathbf{m}}} \rightarrow R_{m, m}=R_{(m) m}
\end{array}
\end{aligned}
$$

where $R_{(i) j}$ is the ith ordinary record in the jth sequence. It may be noted that, $R_{i, i}$ 's are independent random variables, but not necessarily ordered. Let $\mathbf{X}_{\mathbf{S R S}}=$ $\left\{X_{i}, i=1,2, \ldots, m\right\}$ be a SRS of size $m \geq 1$ from a continuous distribution with probability density function (pdf) $f(x)$. Also, let $\mathbf{U}_{\mathbf{R}}=\left(U_{1,1}, U_{2,2}, \ldots, U_{m, m}\right)$ and $\mathbf{L}_{\mathbf{R}}=$ $\left(L_{1,1}, L_{2,2}, \ldots, L_{m, m}\right)$ be the upper and lower RRSS , respectively. Then the density and $\mathrm{cdf}$ of $U_{i, i}$ which are denoted by $f_{i, i}(x)$ and $F_{i, i}(x)$, respectively, are given by (see for instance Arnold et al., 1998)

$$
f_{i, i}(x)=\frac{\{-\ln \bar{F}(x)\}^{i-1}}{(i-1) !} f(x)
$$

$$
F_{i, i}(x)=1-\bar{F}(x) \sum_{t=0}^{i-1} \frac{\{-\ln \bar{F}(x)\}^{t}}{t !}=1-\frac{\Gamma(i ;-\ln \bar{F}(x))}{\Gamma(i)}
$$

where $\bar{F}()=.1-F($.$) and \Gamma(a ; x)$ is known as the incomplete gamma function and is defined as

$$
\Gamma(a ; x)=\int_{x}^{+\infty} u^{a-1} e^{-u} d u, \quad a, x>0 .
$$

The joint density and the survival function of $\mathbf{U}_{\mathbf{R}}$ readily follows:

$$
f_{\mathbf{U}_{\mathbf{R}}}\left(\mathbf{u}_{\mathbf{r}}\right)=\prod_{i=1}^{m} \frac{\left\{-\ln \bar{F}\left(u_{i, i}\right)\right\}^{i-1}}{(i-1) !} f\left(u_{i, i}\right),
$$

and

$$
\begin{aligned}
\bar{F}_{\mathbf{U}_{\mathbf{R}}}\left(\mathbf{u}_{\mathbf{r}}\right) & =\prod_{i=1}^{m} \bar{F}\left(u_{i, i}\right) \sum_{t=0}^{i-1} \frac{\left\{-\ln \bar{F}\left(u_{i, i}\right)\right\}^{t}}{t !} \\
& =\prod_{i=1}^{m} \frac{\Gamma\left(i ;-\ln \bar{F}\left(u_{i, i}\right)\right)}{\Gamma(i)}
\end{aligned}
$$


where $\mathbf{u}_{\mathbf{r}}=\left(u_{1,1}, u_{2,2}, \ldots, u_{m, m}\right)$ is the observed value of $\mathbf{U}_{\mathbf{R}}$. By substituting $\bar{F}$ by $F$ into the Eqs.(3) and (4), the joint density and survival function of lower RRSS are obtained. For an application of proposed plan, we consider a parallel repairable system with minimal repairs, include of $m$ identical components with cdf $F$ that works independently. The minimal system means that the age of system is not changed as a result of the repair. Assume that the ith component $(i=1, \ldots, m)$ can be repaired $i-1$ times $(i \geq 1)$, i.e, it isn't repairable after the $i$ th its failure. Hence, the $\frac{m(m+1)}{2}$ th failure is endangers for the system and the lifetime of the system is given by $\max \left\{T_{1}, \ldots, T_{m}\right\}$, where $T_{i}$ is the lifetime of the ith component. Consequently, in proposed plan $T_{i}$ is identical in distribution with $U_{i, i}$. While system's lifetime is computed according to $\max \left\{U_{1,1}, \ldots, U_{m, m}\right\}$, it will be appropriate to know each $U_{i, i}$ to acquire the entire lifetime system. The information measures for record values have been investigated by several authors, including, Zahedi and Shakil (2006), Baratpour et al. (2007), and Madadi and Tata (2011). Recently, Jafari Jozani and Ahmadi (2014) studied uncertainty and information properties of RSS. Tahmasebi and Jafari(2015) obtained information measures of RSS in Farlie-Gumbel-Morgenstern family. In this paper, we study the information measures such as Shannon's entropy, Rényi entropy, and Kullback-Leibler(KL) information of RRSS data. The organization of this article is as follows. In Section 2, we obtain the Shannon entropies of RRSS and SRS data of the same size in the uniform, exponential, Weibull, Pareto, and gamma distributions. We show that the difference between the Shannon entropy of RRSS and SRS is depends on the parent distribution $F$. In Section 3, similar results with numerical values are derived under the Rényi entropy in uniform and exponential distributions. In Section 4, we show that the KL information between the distribution of $\mathbf{X}_{\mathbf{S R S}}$ and distribution of $\mathbf{U}_{\mathbf{R}}$ is distribution -free and increases as the set size increases.

\section{Shannon Entropy of RRSS}

Shannon (1948) introduced the concepts of entropy and mutual information from communication theory. Entropy is defined as a measure of uncertainty or randomness of a random phenomenon. For a continuous random variable $X$ with pdf $f(x)$, Shannon entropy is defined as

$H(X)=-\int_{-\infty}^{+\infty} f(x) \ln f(x) d x=-\int_{0}^{1} \ln f\left(F^{-1}(u)\right) d u$.

We refer the reader to Cover and Thomas(1991) and the references therein for more details. The Shannon entropy of $\mathbf{X}_{\mathbf{S R S}}$ is given by

$$
H\left(\mathbf{X}_{\mathbf{S R S}}\right)=-\sum_{j=1}^{m} \int f\left(x_{j}\right) \ln f\left(x_{j}\right) d x_{j}=m H\left(X_{1}\right) .
$$

Let us assume that $U_{i, i}$ 's are not necessarily ordered, so by (3) and (5) we have

$$
H\left(\mathbf{U}_{\mathbf{R}}\right)=-\sum_{i=1}^{m} \int f_{i, i}(x) \ln f_{i, i}(x) d x=\sum_{i=1}^{m} H\left(U_{i, i}\right) .
$$

where $H\left(U_{i, i}\right)$ is the entropy of the ith upper record value in the ith sequence. Baratpour et al.(2007) obtained an expression for $H\left(U_{i, i}\right)$ which is given by

$$
\begin{aligned}
H\left(U_{i, i}\right) & =\sum_{k=1}^{i-1}\left(\ln k-\frac{i-1}{k}\right)+(i-1) \gamma-\phi_{f}(i-1) \\
& =H\left(U_{i, i}^{*}\right)-i-E\left[\ln \left(f\left(F^{-1}\left(1-e^{-U_{i, i}^{*}}\right)\right)\right)\right],
\end{aligned}
$$

where

$$
\phi_{f}(i-1)=\int_{0}^{+\infty} \frac{z^{i-1}}{(i-1) !} e^{-z} \ln \left(f\left(F^{-1}\left(1-e^{-z}\right)\right)\right) d z,
$$

and $U_{i, i}^{*}$ is the ith upper record value from gamma distribution with parameters $i$ and 1. By (7) and (8) we have

$$
\begin{aligned}
H\left(\mathbf{U}_{\mathbf{R}}\right) & =\sum_{i=1}^{m} H\left(U_{i, i}\right) \\
& =\sum_{i=1}^{m} H\left(U_{i, i}^{*}\right)-\frac{m(m+1)}{2}-\sum_{i=1}^{m} \phi_{f}(i-1) .
\end{aligned}
$$

Zahedi and Shakil (2006) presented a simple expression of Eq.(8) as

$$
\begin{aligned}
H\left(U_{i, i}\right) & =\ln (\Gamma(i))-(i-1) \psi(i) \\
& -\frac{1}{\Gamma(i)} \int_{-\infty}^{+\infty}[-\ln (1-F(x))]^{i-1} f(x) \ln f(x) d x,
\end{aligned}
$$

where $\psi(i)=\Gamma^{\prime}(i) / \Gamma(i)$ is the digamma function. Now, by using (10) a simple expression for $H\left(\mathbf{U}_{\mathbf{R}}\right)$ is obtained as

$$
H\left(\mathbf{U}_{\mathbf{R}}\right)=\sum_{i=1}^{m}[\ln (\Gamma(i))-(i-1) \psi(i)]-\sum_{i=1}^{m} \phi_{f}(i-1) .
$$

In the sequel, we quantify the difference between $H\left(\mathbf{U}_{\mathbf{R}}\right)$ and $H\left(\mathbf{X}_{\mathbf{S R S}}\right)$. To this end, by using (11), we have

$$
\begin{aligned}
\Delta_{m}(i) & =H\left(\mathbf{U}_{\mathbf{R}}\right)-H\left(\mathbf{X}_{\mathbf{S R S}}\right) \\
& =\sum_{i=1}^{m}[\ln (\Gamma(i))-(i-1) \psi(i)]
\end{aligned}
$$




$$
\begin{aligned}
+ & \sum_{i=1}^{m} \int_{0}^{+\infty}\left[\frac{z^{i-1}}{(i-1) !}-1\right] \\
& \times e^{-z} \ln \left(f\left(F^{-1}\left(1-e^{-z}\right)\right)\right) d z,
\end{aligned}
$$

where $\Delta_{m}(i)$ is depends on the parent distribution $F$. In the following examples, we compare the information content of RRSS with a simple random sample data.

Example 2.1. Suppose $X \sim \operatorname{Uniform}(0,1)$. Then we have

$$
H\left(\tilde{U}_{i, i}\right)=\ln (\Gamma(i))-(i-1) \psi(i),
$$

where $\tilde{U}_{i, i}$ is called ith upper record value in the ith sequence from uniform distribution on $(0,1)$. Let us denote $\tilde{\mathbf{U}}_{\mathbf{R}}=\left(\tilde{U}_{1,1}, \tilde{U}_{2,2}, \ldots, \tilde{U}_{m, m}\right)$. We consider two cases of the RRSS where $m=2$ and $m=3$, respectively. Our calculations show that

$$
H\left(\tilde{\mathbf{U}}_{\mathbf{R}}\right)=\gamma-1, \quad H\left(\tilde{\mathbf{U}}_{\mathbf{R}}\right)=3 \gamma+\ln 2-4,
$$

where $\gamma=-\psi(1)=0.57721566$ is Euler's constant. Now, for two cases, we have

$$
H\left(\tilde{\mathbf{U}}_{\mathbf{R}}\right)<H\left(\mathbf{X}_{\mathbf{S R S}}\right)=0 .
$$

Also, for $m>2$, we obtain

$$
\begin{aligned}
H\left(\tilde{\mathbf{U}}_{\mathbf{R}}\right) & =\sum_{i=1}^{m}[\ln (\Gamma(i))-(i-1) \psi(i)] \\
& =-\sum_{i=1}^{m-1} i \psi(i)+\sum_{i=1}^{m-2} \ln [(i+1) !]-(m-1)<0 .
\end{aligned}
$$

Tabela 1: The values of $H\left(\tilde{\mathbf{U}}_{\mathbf{R}}\right)$ for $m=3$ up to 10 .

\begin{tabular}{|l|cccccccc|}
\hline $\mathrm{m}$ & 3 & 4 & 5 & 6 & 7 & 8 & 9 & 10 \\
\hline$H\left(\tilde{\mathbf{U}}_{\mathbf{R}}\right)$ & -1.5 & -3.5 & -6.3 & -10.1 & -14.7 & -20.3 & -26.9 & -34.3 \\
\hline
\end{tabular}

Table 1 shows the numerical values of $H\left(\tilde{\mathbf{U}}_{\mathbf{R}}\right)$ for $m \in\{3,4, \ldots, 10\}$. From Table 1 , it is observed that $H\left(\tilde{\mathbf{U}}_{\mathbf{R}}\right)<0$ and decreases as $m$ increases. So if $X$ has an uniform distribution on $(0,1)$, then the record ranked set sampling provides the amount of the uncertainty less than simple random sampling.

Remark 2.1. Another expression for $H\left(\mathbf{U}_{\mathbf{R}}\right)$, is given by

$$
H\left(\mathbf{U}_{\mathbf{R}}\right)=H\left(\tilde{\mathbf{U}}_{\mathbf{R}}\right)-\sum_{i=1}^{m} \phi_{f}(i-1)
$$

where $H\left(\tilde{\mathbf{U}}_{\mathbf{R}}\right)$ is defined in (14).

Remark 2.2. Suppose $X \sim \operatorname{Uniform}(\alpha, \beta)$. Then we have

$$
H\left(\mathbf{U}_{\mathbf{R}}\right)=H\left(\tilde{\mathbf{U}}_{\mathbf{R}}\right)+H\left(\mathbf{X}_{\mathbf{S R S}}\right)
$$

$$
\begin{aligned}
= & \sum_{i=1}^{m} \sum_{k=1}^{i-1}\left(\ln k-\frac{i-1}{k}\right)+\gamma\left(\frac{m(m-1)}{2}\right) \\
& +m \ln (\beta-\alpha)<H\left(\mathbf{X}_{\mathbf{S R S}}\right) .
\end{aligned}
$$

Example 2.2. Suppose $X$ has an exponential distribution with pdf $f(x)=\frac{1}{\theta} e^{\frac{-x}{\theta}}$. By using (8), the Shannon entropy of $U_{i, i}$ for $i \geq 2$ is obtained as

$$
\begin{aligned}
H\left(U_{i, i}\right) & =\ln (\Gamma(i))-(i-1) \psi(i)+\ln \theta+i \\
& =H\left(\tilde{U}_{i, i}\right)+\ln \theta+i \\
& =H\left(\tilde{U}_{i, i}\right)+H(X)+i-1,
\end{aligned}
$$

where $H(X)=\ln \theta+1$. We consider two cases where $m=2$ and $m=3$. For $m=2$, we obtain

$$
H\left(\mathbf{X}_{\mathbf{S R S}}\right)=2[1+\ln \theta], \quad H\left(\mathbf{U}_{\mathbf{R}}\right)=2[1+\ln \theta]+\gamma,
$$

Also, for $m=3$, we have

$$
\begin{aligned}
H\left(\mathbf{X}_{\mathbf{S R S}}\right)=3[1+\ln \theta], & H\left(\mathbf{U}_{\mathbf{R}}\right) \\
& =3[1+\ln \theta]+2 \gamma+(\ln 2-1) .
\end{aligned}
$$

Now, for two cases, we have

$$
H\left(\mathbf{U}_{\mathbf{R}}\right)-H\left(\mathbf{X}_{\mathbf{S R S}}\right)>0 .
$$

Hence, for $m \geq 2$, we obtain

$$
\begin{aligned}
H\left(\mathbf{U}_{\mathbf{R}}\right)= & \sum_{i=1}^{m} H\left(U_{i, i}\right)=\sum_{i=1}^{m}\left[H\left(\tilde{U}_{i, i}\right)+H(X)+i-1\right] \\
= & H\left(\tilde{\mathbf{U}}_{\mathbf{R}}\right)+m H(X)+\frac{m(m+1)}{2}-m \\
= & -\sum_{i=1}^{m-1} i \psi(i)+\sum_{i=1}^{m-2} \ln [(i+1) !] \\
& +\frac{(m-2)(m-1)}{2}+H\left(\mathbf{X}_{\mathbf{S R S}}\right)
\end{aligned}
$$

In the sequel, we have

$$
\begin{aligned}
H\left(\mathbf{U}_{\mathbf{R}}\right)-H\left(\mathbf{X}_{\mathbf{S R S}}\right)= & -\sum_{i=1}^{m-1} i \psi(i)+\sum_{i=1}^{m-2} \ln [(i+1) !] \\
& +\frac{(m-2)(m-1)}{2}=k(m)
\end{aligned}
$$

By noting that $\psi(i)=-\gamma+\sum_{j=1}^{m-1}\left(j^{-1}\right)$, for $m \geq 2$. We can easily find the following

$k(m+1)-k(m)=m\left[\gamma-\sum_{j=2}^{m-1}\left(j^{-1}\right)\right]+\ln (m !)-1=w(m)$.

Note that for all $m \geq 2, w(m)>0$. This implies that $k(m)$ increases as $m$ increase. Table 2 shows the values of $k(m)$ for $m \in\{2,3, \ldots, 10\}$. From Table 2, it is observed that if $X$ has an exponential distribution with mean $\theta$, then the record ranked set sampling provides the amount of the uncertainty more than simple random sampling. 
Tabela 2- The values of $k(m)$ for $m=2$ up to 10

\begin{tabular}{l|ccccccccc}
\hline $\mathrm{m}$ & 2 & 3 & 4 & 5 & 6 & 7 & 8 & 9 & 10 \\
\hline
\end{tabular}

\begin{tabular}{l|lllllllll}
\hline$k(m)$ & 0.57 & 0.87 & 2.02 & 4.15 & 7.25 & 11.34 & 16.41 & 22.47 & 29.53 \\
\hline
\end{tabular}

Example 2.3. Suppose $X$ has a Pareto distribution with pdf $f(x)=\theta x^{-(\theta+1)} I_{(1, \infty)}(x)$. By using (8), the Shannon entropy of $U_{i, i}$ for $i \geq 2$ is obtained as

$$
H\left(U_{i, i}\right)=H\left(\tilde{U}_{i, i}\right)+H(X)+(i !-1) \frac{\theta+1}{\theta},
$$

where $H(X)=-\ln (\theta)+\left(\frac{\theta+1}{\theta}\right)$. We consider two cases where $m=2$ and $m=3$. For $m=2$, we obtain

$$
\begin{gathered}
H\left(\mathbf{X}_{\mathbf{S R S}}\right)=2\left[-\ln \theta+\frac{\theta+1}{\theta}\right], \\
H\left(\mathbf{U}_{\mathbf{R}}\right)=\frac{3}{\theta}-2 \ln \theta+\gamma+2 .
\end{gathered}
$$

Also, for $m=3$, we have

$$
\begin{array}{r}
H\left(\mathbf{X}_{\mathbf{S R S}}\right)=3\left[-\ln \theta+\frac{\theta+1}{\theta}\right], \\
H\left(\mathbf{U}_{\mathbf{R}}\right)=\frac{9}{\theta}-3 \ln \theta+3 \gamma+\ln 2+4 .
\end{array}
$$

Now, for two cases, we have

$$
H\left(\mathbf{U}_{\mathbf{R}}\right)-H\left(\mathbf{X}_{\mathbf{S R S}}\right)>0 .
$$

Also, for $m \geq 2$, we obtain

$$
\begin{aligned}
H\left(\mathbf{U}_{\mathbf{R}}\right)= & \sum_{i=1}^{m} H\left(U_{i, i}\right) \\
= & \sum_{i=1}^{m}\left[H\left(\tilde{U}_{i, i}\right)+H(X)+(i !-1) \frac{\theta+1}{\theta}\right] \\
= & H\left(\tilde{\mathbf{U}}_{\mathbf{R}}\right)+m H(X)+\frac{\theta+1}{\theta}\left[\sum_{i=1}^{m} i !-m\right] \\
= & -\sum_{i=1}^{m-1} i \psi(i)+\sum_{i=1}^{m-2} \ln [(i+1) !] \\
& -(m-1)+\frac{\theta+1}{\theta}\left[\sum_{i=1}^{m} i !-m\right]+H\left(\mathbf{X}_{\mathbf{S R S}}\right) .
\end{aligned}
$$

Finally, numerical computations indicate that

$$
\begin{aligned}
D(m, \theta)= & H\left(\mathbf{U}_{\mathbf{R}}\right)-H\left(\mathbf{X}_{\mathbf{S R S}}\right) \\
= & -\sum_{i=1}^{m-1} i \psi(i)+\sum_{i=1}^{m-2} \ln [(i+1) !] \\
& -(m-1)+\frac{\theta+1}{\theta}\left[\sum_{i=1}^{m} i !-m\right]>0 .
\end{aligned}
$$

Table 3 shows the values of $D(m, \theta)$ for $\theta=2$ and
Tabela 3: The values of $D(m, \theta)$ for $m=3$ up to 10

Tabela 3: The values of $D(m, \theta)$ for $m=3$ up to 10

\begin{tabular}{l|cccccccc}
\hline $\mathrm{m}$ & 3 & 4 & 5 & 6 & 7 & 8 & 9 & 10 \\
\hline$D(m, \theta)$ & 7.4 & 39.9 & 215.6 & 1290.3 & 8844.2 & 69317.1 & 613629.1 & 6056820 \\
\hline
\end{tabular}

$m \in\{3,4, \ldots, 10\}$. Also, $D(m, \theta)$ is an increasing function of $m$, if $\theta$ is fixed. So if $X$ has a Pareto distribution with parameter $\theta$, then the record ranked set sampling provides the amount of the uncertainty more than simple random sampling.

Example 2.4. Suppose $X$ has a Weibull with pdf $f(x)=$ $\lambda \beta x^{\beta-1} \exp \left(-\lambda x^{\beta}\right)$. By using (8), the Shannon entropy of $U_{i, i}$ for $i \geq 2$ is obtained as

$$
H\left(U_{i, i}\right)=H\left(\tilde{U}_{i, i}\right)+H(X)+\left(\frac{1-\beta}{\beta}\right) \sum_{k=1}^{i-1} \frac{1}{k}+i-1,
$$

where $H(X)=-\ln \left(\lambda^{\frac{1}{\beta}} \beta\right)+\left(\frac{1-\beta}{\beta}\right) \psi(1)+1$. We consider two cases where $m=2$ and $m=3$. For $m=2$, we obtain

$$
H\left(\mathbf{X}_{\mathbf{S R S}}\right)=2 H(X), H\left(\mathbf{U}_{\mathbf{R}}\right)=2 H(X)+\gamma+\frac{1-\beta}{\beta}
$$

Also, for $m=3$, we have

$H\left(\mathbf{X}_{\mathbf{S R S}}\right)=3 H(X), H\left(\mathbf{U}_{\mathbf{R}}\right)=3 H(X)+3 \gamma+\ln 2-\frac{7}{2}+\frac{5}{2 \beta}$.

Now , for two cases, we have

$$
H\left(\mathbf{U}_{\mathbf{R}}\right)-H\left(\mathbf{X}_{\mathbf{S R S}}\right)<0, \text { for } \beta>2 .
$$

Finally, for $m \geq 2$, we obtain

$$
\begin{aligned}
H\left(\mathbf{U}_{\mathbf{R}}\right)= & \sum_{i=1}^{m} H\left(U_{i, i}\right)=\sum_{i=1}^{m} H\left(\tilde{U}_{i, i}\right)+m H(X) \\
& +\left(\frac{1-\beta}{\beta}\right) \sum_{i=2}^{m} \sum_{k=1}^{i-1} \frac{1}{k}+\frac{(m+2)(m-1)}{2} \\
= & H\left(\tilde{\mathbf{U}}_{\mathbf{R}}\right)+H\left(\mathbf{X}_{\mathbf{S R S}}\right)+\left(\frac{1-\beta}{\beta}\right) \sum_{i=2}^{m} \sum_{k=1}^{i-1} \frac{1}{k} \\
& +\frac{(m+2)(m-1)}{2} \\
= & -\sum_{i=1}^{m-1} i \psi(i)+\sum_{i=1}^{m-2} \ln [(i+1) !] \\
& +H\left(\mathbf{X}_{\mathbf{S R S}}\right)+\left(\frac{1-\beta}{\beta}\right) \sum_{i=2}^{m} \sum_{k=1}^{i-1} \frac{1}{k}+\frac{(m)(m-1)}{2} .
\end{aligned}
$$

In the sequel, the difference between $H\left(\mathbf{U}_{\mathbf{R}}\right)$ and $H\left(\mathbf{X}_{\mathbf{S R S}}\right)$ is given by

$$
\delta(m, \beta)=-\sum_{i=1}^{m-1} i \psi(i)+\sum_{i=1}^{m-2} \ln [(i+1) !]
$$




$$
\begin{aligned}
& +\left(\frac{1-\beta}{\beta}\right) \sum_{i=2}^{m} \sum_{k=1}^{i-1} \frac{1}{k}+\frac{(m)(m-1)}{2}<0, \\
& \quad \text { for } \beta>2 .
\end{aligned}
$$

Table 4 shows the values of $\delta(m, \beta)$ for $\beta=3$ and $m \in\{3,4, \ldots, 10\}$. Numerical computations indicate that $\delta(m, \beta)$ is decreasing function of $m$, if $\beta$ is fixed. So if $X$ has a Weibull distribution with parameters $(\lambda, \beta)$, then for $\beta>2$ the record ranked set sampling provides the amount of the uncertainty less than simple random sampling.

Tabela 4: The values of $\delta(m, \beta)$ for $m=3$ up to 10

\begin{tabular}{|l|cccccccc|}
\hline $\mathrm{m}$ & 3 & 4 & 5 & 6 & 7 & 8 & 9 & 10 \\
\hline$\delta(m, \beta)$ & -0.24 & -0.44 & -0.67 & -0.94 & -1.23 & -1.54 & -1.87 & -2.22 \\
\hline
\end{tabular}

Example 2.5. Let $U_{i, i}^{*}$ be the i-th upper record value from $\operatorname{Gamma}(i, 1)$, then the Shannon entropy of $\mathbf{U}_{\mathbf{R}}^{*}=$ $\left(U_{1,1}^{*}, U_{2,2}^{*}, \ldots, U_{m, m}^{*}\right)$ is obtained as

$$
\begin{aligned}
H\left(\mathbf{U}_{\mathbf{R}}^{*}\right) & =\sum_{i=1}^{m} H\left(U_{i, i}^{*}\right)=\sum_{i=1}^{m}[\ln (\Gamma(i))-(i-1) \psi(i)+i] \\
& =-\sum_{i=1}^{m-1} i \psi(i)+\sum_{i=1}^{m-2} \ln [(i+1) !]+\frac{m^{2}-m+2}{2} \\
& =H\left(\mathbf{X}_{\text {SRS }}\right) .
\end{aligned}
$$

Lemma 2.1. If $f(x)$ is increasing in $x$, then $H\left(\mathbf{U}_{\mathbf{R}}\right)$ is decreasing in $m$.

Proof. By using (15), we have

$$
\begin{aligned}
d_{1}(m)= & H\left(\mathbf{U}_{\mathbf{R}}^{(\mathbf{m}+\mathbf{1})}\right)-H\left(\mathbf{U}_{\mathbf{R}}^{(\mathbf{m})}\right) \\
= & H\left(\tilde{\mathbf{U}}_{\mathbf{R}}^{(\mathbf{m}+\mathbf{1})}\right)-H\left(\tilde{\mathbf{U}}_{\mathbf{R}}^{(\mathbf{m})}\right) \\
& -\sum_{i=1}^{m+1} \phi_{f}(i-1)+\sum_{i=1}^{m} \phi_{f}(i-1) \\
= & \ln (m !)-m \psi(m)-\phi_{f}(m) \\
= & \ln (m !)-m \psi(m) \\
& -\int_{0}^{+\infty} \frac{z^{m}}{(m) !} e^{-z} \ln \left(f\left(F^{-1}\left(1-e^{-z}\right)\right)\right) d z \\
< & 0 .
\end{aligned}
$$

Thus the proof is complete.

Baratpur et al.(2007) obtained an upper bound for $H\left(U_{i, i}\right)$ which is depends on the hazard rate function $r()=.\frac{f(.)}{\bar{F}(.)}$ as follows:

$$
H\left(U_{i, i}\right) \leq H\left(U_{i, i}^{*}\right)-i-B_{i} I(A),
$$

where $B_{i}=\frac{(i-1)^{(i-1)} e^{-(i-1)}}{(i-1) !}, I(A)=\int_{A} r(y) \ln f(y) d y$ and $A=\{y \mid f(y) \leq 1\}$. Now, by using (15) and (25) an upper bound for $H\left(\mathbf{U}_{\mathbf{R}}\right)$ is given by

$$
H\left(\mathbf{U}_{\mathbf{R}}\right) \leq H\left(\tilde{\mathbf{U}}_{\mathbf{R}}\right)-\sum_{j=o}^{m+1} \frac{j^{j} e^{-j}}{j !} I(A) .
$$

Remark 2.3. Similar results for $H\left(\mathbf{U}_{\mathbf{R}}\right)$ which given in this section, can be obtained for $H\left(\mathbf{L}_{\mathbf{R}}\right)$.

Rao et al. (2004) introduced a new measure of information that extends the Shannon entropy to continuous random variables, and called it cumulative residual entropy (CRE). The CRE is based on survival function $\bar{F}(x)$, and is defined as

$$
\mathcal{E}(X)=-\int_{0}^{+\infty} \bar{F}(x) \ln \bar{F}(x) d x .
$$

The CRE of $\mathbf{X}_{\mathbf{S R S}}$ and $\mathbf{U}_{\mathbf{R}}$ are obtained as

$\mathcal{E}\left(\mathbf{X}_{\mathbf{S R S}}\right)=n \mathcal{E}(X) \mu^{n-1}, \mathcal{E}\left(\mathbf{U}_{\mathbf{R}}\right)=\sum_{i} \mathcal{E}\left(U_{(i, i)}\right) \prod_{j \neq i} \mu_{(j, j)}$,

where $\mu=\int_{0}^{+\infty} \bar{F}(x) d x$ and $\mu_{(j, j)}=\int_{0}^{+\infty} \bar{F}_{j, j}(x) d x$.

\section{Rényi entropy of RRSS}

In information theory, the Rényi entropy is a generalization for the Shannon entropy. The Rényi entropy of order $\lambda$ is defined as

$$
H_{\lambda}(X)=\frac{1}{1-\lambda} \ln \int_{-\infty}^{+\infty} f^{\lambda}(x) d x,
$$

where $\lambda>0, \lambda \neq 1$, and $H(X)=\lim _{\lambda \rightarrow 1} H_{\lambda}(X)=$ $-\int_{-\infty}^{\infty} f(x) \ln f(x) d x$ is the Shannon entropy if both integrals exist(Rényi, 1961). Rényi information is much more flexible than the Shannon entropy due to the parameter $\lambda$. It is an important measure in various applied sciences such as statistics (Song, 2001), ecology (Harte, 2011), engineering (Lenzi et.al., 2000), and economics (Ullah, 1988). In this section, we obtain the Rényi entropy of $\mathbf{U}_{\mathbf{R}}$ and compare its with the Rényi entropy of $\mathbf{X}_{\text {SRS }}$. To this end, it is easy to show that the Rényi entropy of a $S R S$ of size $m$ from $f$ is given by

$$
H_{\lambda}\left(\mathbf{X}_{\mathbf{S R S}}\right)=\sum_{i=1}^{m} H_{\lambda}\left(X_{i}\right)=m H\left(X_{1}\right) .
$$

Also, for a RRSS of size $m$, we have

$$
H_{\lambda}\left(\mathbf{U}_{\mathbf{R}}\right)=\sum_{i=1}^{m} H_{\lambda}\left(U_{i, i}\right),
$$

where $H_{\lambda}\left(U_{i, i}\right)$ is the Rényi entropy of the ith upper record value in the ith sequence. Abbasnejad and Arghami (2011) obtained an expression for $H_{\lambda}\left(U_{i, i}\right)$ as

$$
H_{\lambda}\left(U_{i, i}\right)=H_{\lambda}\left(U_{i, i}^{*}\right)-\left(\frac{\lambda(i-1)+1}{\lambda-1}\right) \ln (\lambda)
$$




$$
-\frac{1}{\lambda-1} \ln E\left[f^{\lambda-1}\left(F^{-1}\left(1-e^{-V_{i, i}}\right)\right)\right],
$$

where $U_{i, i}^{*} \sim \operatorname{Gamma}(i, 1)$ denotes ith upper record of standard exponential distribution and $V_{i, i} \sim \operatorname{Gamma}(\lambda(i-$ $1)+1,1)$. Also, the Rényi entropy of $U_{i, i}^{*}$ is given by

$$
\begin{aligned}
H_{\lambda}\left(U_{i, i}^{*}\right)= & \frac{\lambda}{\lambda-1} \ln (\Gamma(i))-\frac{1}{\lambda-1} \ln (\Gamma(\lambda(i-1)+1)) \\
& +\frac{\lambda(i-1)+1}{\lambda-1} \ln (\lambda) .
\end{aligned}
$$

By using (31) and (33) an expression for $H_{\lambda}\left(\mathbf{U}_{\mathbf{R}}\right)$ is obtained as

$$
\begin{aligned}
H_{\lambda}\left(\mathbf{U}_{\mathbf{R}}\right)= & \sum_{i=1}^{m} H_{\lambda}\left(U_{i, i}^{*}\right)-\sum_{i=1}^{m}\left[\left(\frac{\lambda(i-1)+1}{\lambda-1}\right) \ln (\lambda)\right] \\
& -\frac{1}{\lambda-1} \sum_{i=1}^{m} \ln \int_{0}^{+\infty} \frac{v^{\lambda(i-1)} e^{-v}}{\Gamma(\lambda(i-1)+1)} \\
& \times f^{\lambda-1}\left(F^{-1}\left(1-e^{-v}\right)\right) d v \\
= & \frac{\lambda}{\lambda-1} \sum_{i=1}^{m} \ln \Gamma(i) \\
& -\frac{1}{\lambda-1} \sum_{i=1}^{m} \ln \Gamma(\lambda(i-1)+1) \\
& -\frac{1}{\lambda-1} \sum_{i=1}^{m} \ln \int_{0}^{+\infty} \frac{v^{\lambda(i-1)} e^{-v}}{\Gamma(\lambda(i-1)+1)} \\
& \times f^{\lambda-1}\left(F^{-1}\left(1-e^{-v}\right)\right) d v \\
= & H_{\lambda}\left(\tilde{\mathbf{U}}_{\mathbf{R}}\right) \\
& -\frac{1}{\lambda-1} \sum_{i=1}^{m} \ln E\left[f^{\lambda-1}\left(F^{-1}\left(1-e^{-V_{i, i}}\right)\right)\right],
\end{aligned}
$$

where $H_{\lambda}\left(\tilde{\mathbf{U}}_{\mathbf{R}}\right)$ is the Rényi entropy of $\tilde{\mathbf{U}}_{\mathbf{R}}$ from uniform distribution on $(0,1)$. It can be easily shown that

$$
H_{\lambda}\left(\tilde{\mathbf{U}}_{\mathbf{R}}\right)=\frac{\lambda}{\lambda-1} \sum_{i=1}^{m} \ln \left[\frac{\Gamma(i)}{(\Gamma(\lambda(i-1)+1))^{\frac{1}{\lambda}}}\right] .
$$

Table 5 shows the numerical values of $H_{\lambda}\left(\tilde{\mathbf{U}}_{\mathbf{R}}\right)$ for $m \in$ $\{2,3, \ldots, 10\}$ and two cases $0<\lambda<1$ and $\lambda>1$. From Table 3 , it is observed that $H_{\lambda}\left(\tilde{\mathbf{U}}_{\mathbf{R}}\right)<0$ and decreases as $m$ increases. So if $X$ has an uniform distribution on $(0,1)$, then the record ranked set sampling provides the amount of the Rényi entropy less than simple random sampling. We can show that the difference between $H_{\lambda}\left(\mathbf{U}_{\mathbf{R}}\right)$ and $H_{\lambda}\left(\mathbf{X}_{\mathbf{S R S}}\right)$ is depends on the parent distribution $F$. To this end, by using (34) we have

$$
\begin{aligned}
\delta_{m}^{\lambda}(i)= & H_{\lambda}\left(\mathbf{U}_{\mathbf{R}}\right)-H_{\lambda}\left(\mathbf{X}_{\mathbf{S R S}}\right) \\
= & \frac{\lambda}{\lambda-1} \sum_{i=1}^{m} \ln \left[\frac{\Gamma(i)}{(\Gamma(\lambda(i-1)+1))^{\frac{1}{\lambda}}}\right] \\
& -\frac{1}{\lambda-1}
\end{aligned}
$$

Tabela 5: The numerical values of $H_{\lambda}\left(\tilde{\mathbf{U}}_{\mathbf{R}}\right)$

\begin{tabular}{c|cccc|cccc}
\hline & \multicolumn{4}{|c|}{$0<\lambda<1$} & \multicolumn{4}{c}{$\lambda>1$} \\
\hline$m$ & 0.2 & 0.4 & 0.6 & 0.8 & 2 & 4 & 6 & 8 \\
\hline 2 & -0.10 & -0.19 & -0.28 & -0.35 & -0.69 & -1.05 & -1.31 & -1.51 \\
3 & -0.32 & -0.58 & -0.79 & -0.98 & -1.79 & -2.61 & -3.16 & -3.58 \\
4 & -0.58 & -1.03 & -1.39 & -1.70 & -2.99 & -4.27 & -5.12 & -5.77 \\
5 & -0.88 & -1.52 & -2.03 & -2.46 & -4.24 & -5.98 & -7.14 & -8.01 \\
6 & -1.19 & -2.03 & -2.70 & -3.25 & -5.52 & -7.72 & -9.18 & -10.28 \\
7 & -1.52 & -2.56 & -3.38 & -4.06 & -6.82 & -9.48 & -11.24 & -12.57 \\
8 & -1.86 & -3.10 & -4.07 & -4.88 & -8.14 & -11.26 & -13.32 & -14.87 \\
9 & -2.20 & -3.65 & -4.78 & -5.71 & -9.46 & -13.04 & -15.40 & -17.19 \\
10 & -2.55 & -4.21 & -5.49 & -6.55 & -10.79 & -14.83 & -17.50 & -19.50 \\
\hline
\end{tabular}

$$
\times \sum_{i=1}^{m} \ln \left[\frac{\int_{0}^{+\infty} \frac{v^{\lambda(i-1)}}{\Gamma(\lambda(i-1)+1)} e^{-v} f^{\lambda-1}\left(F^{-1}\left(1-e^{-v}\right)\right) d v}{\int_{0}^{+\infty} e^{-v} f^{\lambda-1}\left(F^{-1}\left(1-e^{-v}\right)\right) d v}\right] .
$$

Remark 3.1. Suppose $X \sim$ Uniform $(\alpha, \beta)$. Then we have

$$
H_{\lambda}\left(\mathbf{U}_{\mathbf{R}}\right)=H_{\lambda}\left(\tilde{\mathbf{U}}_{\mathbf{R}}\right)+m \ln (\beta-\alpha)<H_{\lambda}\left(\mathbf{X}_{\mathbf{S R S}}\right) .
$$

Example 3.1. Suppose $X$ has an exponential distribution with pdf $f(x)=\frac{1}{\theta} e^{\frac{-x}{\theta}}$. By using (34), the Rényi entropy of order $\lambda$ for $\mathbf{U}_{\mathbf{R}}$ is obtained as

$$
\begin{aligned}
H_{\lambda}\left(\mathbf{U}_{\mathbf{R}}\right) & =H_{\lambda}\left(\tilde{\mathbf{U}}_{\mathbf{R}}\right)+m \ln \theta+\frac{\ln \lambda}{\lambda-1}\left[\frac{\lambda m(m-1)}{2}+m\right] \\
& =H_{\lambda}\left(\tilde{\mathbf{U}}_{\mathbf{R}}\right)+H_{\lambda}\left(\mathbf{X}_{\mathbf{S R S}}\right)+\frac{\lambda \ln (\lambda)[m(m-1)]}{2(\lambda-1)},
\end{aligned}
$$

where $H_{\lambda}\left(\mathbf{X}_{\mathbf{S R S}}\right)=m\left[\ln \theta+\frac{\ln \lambda}{\lambda-1}\right]$. Also the difference between $H_{\lambda}\left(\mathbf{U}_{\mathbf{R}}\right)$ and $H_{\lambda}\left(\mathbf{X}_{\mathbf{S R S}}\right)$ is given by

$$
\begin{aligned}
\delta_{m}^{\lambda}(i) & =\frac{\lambda}{\lambda-1} \sum_{i=1}^{m} \ln \left[\frac{\Gamma(i)}{(\Gamma(\lambda(i-1)+1))^{\frac{1}{\lambda}}}\right] \\
& +\frac{\lambda \ln (\lambda)[m(m-1)]}{2(\lambda-1)} .
\end{aligned}
$$

Note that $\delta_{m}^{\lambda}(i)<0$ (i.e. $\left.H_{\lambda}\left(\mathbf{U}_{\mathbf{R}}\right)<H_{\lambda}\left(\mathbf{X}_{\mathbf{S R S}}\right)\right)$ for any $0<\lambda<1$ and all $m \in \mathbb{N}$.

Lemma 3.1. If $f(x)$ is increasing in $x$, then $H_{\lambda}\left(\mathbf{U}_{\mathbf{R}}\right)$ is decreasing in $m$.

Proof. By using (34), we have

$$
\begin{aligned}
\delta_{\lambda}^{*}(m)= & H_{\lambda}\left(\mathbf{U}_{\mathbf{R}}^{(\mathbf{m}+\mathbf{1})}\right)-H_{\lambda}\left(\mathbf{U}_{\mathbf{R}}^{(\mathbf{m})}\right) \\
= & H_{\lambda}\left(\tilde{\mathbf{U}}_{\mathbf{R}}^{(\mathbf{m}+\mathbf{1})}\right)-H_{\lambda}\left(\tilde{\mathbf{U}}_{\mathbf{R}}^{(\mathbf{m})}\right) \\
& -\frac{1}{\lambda-1} \sum_{i=1}^{m+1} \ln E\left[f^{\lambda-1}\left(F^{-1}\left(1-e^{-V_{i, i}}\right)\right)\right]
\end{aligned}
$$




$$
\begin{aligned}
& +\frac{1}{\lambda-1} \sum_{i=1}^{m} \ln E\left[f^{\lambda-1}\left(F^{-1}\left(1-e^{-V_{i, i}}\right)\right)\right] \\
= & \frac{\lambda}{\lambda-1} \ln \left[\frac{\Gamma(m+1)}{(\Gamma(\lambda(m)+1))^{\frac{1}{\lambda}}}\right] \\
& -\frac{1}{\lambda-1} \ln E\left[f^{\lambda-1}\left(F^{-1}\left(1-e^{-V_{m+1, m+1}}\right)\right)\right] \\
< & 0 .
\end{aligned}
$$

Thus the proof is complete.

Abbasnejad and Arghami (2011) presented an upper bound of $H_{\lambda}\left(U_{i, i}\right)$ for any $0<\lambda<1$ as follows:

$$
\begin{aligned}
H_{\lambda}\left(U_{i, i}\right) \leq & H_{\lambda}\left(U_{i, i}^{*}\right)-\frac{1}{\lambda-1}\left[(\lambda(i-1)+1) \ln \lambda+\ln C_{i}\right] \\
& +S_{\lambda}(X)
\end{aligned}
$$

where $C_{i}=\frac{e^{-\lambda(i-1)}(\lambda(i-1))^{\lambda(i-1)}}{\Gamma(\lambda(i-1)+1)}$ and

$S_{\lambda}(X)=-\left(\frac{1}{\lambda-1}\right) \ln \int_{-\infty}^{+\infty} r(x) f^{\lambda-1}(x) d x$. Now, by using (34) and (40) an upper bound of $H_{\lambda}\left(\mathbf{U}_{\mathbf{R}}\right)$ for any $0<$ $\lambda<1$ is given by

$$
H_{\lambda}\left(\mathbf{U}_{\mathbf{R}}\right) \leq H_{\lambda}\left(\tilde{\mathbf{U}}_{\mathbf{R}}\right)-\frac{1}{\lambda-1} \sum_{i=1}^{m} \ln C_{i}+m S_{\lambda}(X) .
$$

Remark 3.2. Similar results with $H_{\lambda}\left(\mathbf{U}_{\mathbf{R}}\right)$ can be obtained for $H_{\lambda}\left(\mathbf{L}_{\mathbf{R}}\right)$.

\section{Kullback-Leibler Information of RRSS}

The Kullback-Leibler (KL)divergence for two random variables $X$ and $Y$ with pdfs $f$ and $g$ is given by

$$
K(X, Y)=\int f(t) \ln \left(\frac{f(t)}{g(t)}\right) d t
$$

The KL divergence measures the distance between two density functions. This divergence is also known as information divergence and relative entropy. For more details see Kullback and Leibler (1951). Using the same idea as in (42), we define the KL between $\mathbf{X}_{\mathbf{S R S}}$ and $\mathbf{U}_{\mathbf{R}}$ as

$$
\begin{aligned}
K\left(\mathbf{X}_{\mathbf{S R S}}, \mathbf{U}_{\mathbf{R}}\right) & =\int f_{\mathbf{X}_{\mathbf{S R S}}}(x) \ln \left(\frac{f_{\mathbf{X}_{\mathbf{S R S}}}(x)}{f_{\mathbf{U}_{\mathbf{R}}}(x)}\right) d x \\
& =\sum_{i=1}^{m} \int f(x) \ln \left(\frac{f(x)}{f_{i, i}(x)}\right) d x \\
& =\sum_{i=1}^{m} K\left(X, U_{i, i}\right) \\
& =\sum_{i=1}^{m} \int f(x) \ln \left[\frac{f(x)}{\frac{\{-\ln \bar{F}(x)\}^{i-1}}{(i-1) !} f(x)}\right] d x \\
& =-\sum_{i=1}^{m} \int_{0}^{1} \ln \left[\frac{\{-\ln (1-u)\}^{i-1}}{(i-1) !}\right] d u
\end{aligned}
$$

$$
\begin{aligned}
& =\sum_{i=1}^{m}[\gamma(i-1)+\ln ((i-1) !)] \\
& =\gamma \frac{m(m-1)}{2}+\sum_{i=1}^{m} \ln ((i-1) !):=c_{m} .
\end{aligned}
$$

Note that $K\left(\mathbf{X}_{\mathbf{S R S}}, \mathbf{U}_{\mathbf{R}}\right)$ is distribution-free, and $\left\{c_{m}, m=\right.$ $1,2, \ldots\}$ is a nondecreasing sequence of non-negative real values for $m \in N$. That is, the KL information between the distribution of SRS and the distribution of RRSS of the same size increases as the sample size $m$ increases.

Remark 4.1. It is well known that the KL divergence is non-symmetric and cannot be considered as a distance metric. In our problem, note that

$$
\begin{aligned}
K\left(\mathbf{U}_{\mathbf{R}}, \mathbf{X}_{\mathbf{S R S}}\right) & =\sum_{i=1}^{m} \int f_{i, i}(x) \ln \left(\frac{f_{i, i}(x)}{f(x)}\right) d x=\sum_{i=1}^{m} K\left(U_{i, i}, X\right) \\
& =-\sum_{i=1}^{m} H\left(\tilde{U}_{i, i}\right) \\
& =-\sum_{i=1}^{m}[\ln (\Gamma(i))-(i-1) \psi(i)] \\
& =\sum_{i=1}^{m-1} i \psi(i)-\sum_{i=1}^{m-2} \ln [(i+1) !]+(m-1) .
\end{aligned}
$$

Also the Kullback-Leibler distance (KLD) between $\mathbf{X}_{\mathbf{S R S}}$ and $\mathbf{U}_{\mathbf{R}}$ is proposed as

$$
\begin{aligned}
K L D\left(\mathbf{X}_{\mathbf{S R S}}, \mathbf{U}_{\mathbf{R}}\right) & =K\left(\mathbf{X}_{\mathbf{S R S}}, \mathbf{U}_{\mathbf{R}}\right)+K\left(\mathbf{U}_{\mathbf{R}}, \mathbf{X}_{\mathbf{S R S}}\right) \\
& =\sum_{i=1}^{m-1} i \psi(i)+(m-1)+\gamma \frac{m(m-1)}{2} .
\end{aligned}
$$

Let $\mathbf{X}_{\mathbf{S R S}}$ be a SRS of size $m$ from $f(x)$ and let $\mathbf{V}_{\mathbf{R}}$ and $\mathbf{Y}_{\text {SRS }}$ be independent RRSS and SRS samples of the same size from another distribution with pdf $g(x)$ and cdf $G(x)$, respectively. Then,

$$
\begin{aligned}
K\left(\mathbf{X}_{\mathbf{S R S}}, \mathbf{V}_{\mathbf{R}}\right)= & \sum_{i=1}^{m} \int f(x) \ln \left(\frac{f(x)}{g_{i, i}(x)}\right) d x \\
= & m \int f(x) \ln \left[\frac{f(x)}{g(x)}\right] d x \\
& -\sum_{i=1}^{m} \int f(x) \ln \left(\frac{[-\ln \bar{G}(x)]^{i-1}}{(i-1) !}\right) d x \\
= & K\left(\mathbf{X}_{\mathbf{S R S}}, \mathbf{Y}_{\mathbf{S R S}}\right)-A_{f, G}(m),
\end{aligned}
$$

where

$$
A_{f, G}(m)=\sum_{i=1}^{m} \int_{0}^{1} f\left(G^{-1}(u)\right) \ln \left(\frac{[-\ln (1-u)]^{i-1}}{(i-1) !}\right) d u
$$




$$
\begin{aligned}
& =\sum_{i=1}^{m} \int_{0}^{\infty} f\left(G^{-1}\left(1-e^{-w}\right)\right) \ln \left[\frac{w^{i-1}}{(i-1) !}\right] e^{-w} d w \\
& =\sum_{i=1}^{m} E\left[\ln \left[\frac{W^{i-1}}{(i-1) !}\right] f\left(G^{-1}\left(1-e^{-W}\right)\right)\right]
\end{aligned}
$$

where $W \sim \exp (1)$. Note that in this case $A_{f, G}(m)$ depends on the density function $X$ and the parent distribution $Y$ samples. Here again $K\left(\mathbf{X}_{\mathbf{S R S}}, \mathbf{Y}_{\mathbf{S R S}}\right) \leq K\left(\mathbf{X}_{\mathbf{S R S}}, \mathbf{V}_{\mathbf{R}}\right)$, if $A_{f, G}(m) \leq 0$.

Another result which is of interest is to compare $K\left(\mathbf{U}_{\mathbf{R}}, \mathbf{V}_{\mathbf{R}}\right)$ and $K\left(\mathbf{X}_{\mathbf{S R S}}, \mathbf{Y}_{\mathbf{S R S}}\right)$. To this end, we have

$$
\begin{aligned}
K\left(\mathbf{U}_{\mathbf{R}}, \mathbf{V}_{\mathbf{R}}\right)= & \sum_{i=1}^{m} \int f_{i, i}(x) \ln \left(\frac{f_{i, i}(x)}{g_{i, i}(x)}\right) d x \\
= & \sum_{i=1}^{m} \int f_{i, i}(x)\left\{\ln \left(\frac{f(x)}{g(x)}\right)\right. \\
& +\ln \left[\frac{(-\ln \bar{F}(x))^{i-1}}{\left.\left.(-\ln \bar{G}(x))^{i-1}\right]\right\} d x}\right. \\
= & \sum_{i=1}^{m} \frac{m(-\ln \bar{F}(x))^{i-1}}{m(i-1) !} \int f(x) \ln \left[\frac{f(x)}{g(x)}\right] d x \\
& +\sum_{i=1}^{m} \int \frac{f(x)\{-\ln \bar{F}(x)\}^{i-1}}{(i-1) !} \\
& \times \ln \left[\frac{(-\ln \bar{F}(x))^{i-1}}{(-\ln \bar{G}(x))^{i-1}}\right] d x \\
= & \sum_{i=1}^{m} \frac{(-\ln \bar{F}(x))^{i-1}}{m(i-1) !} K\left(\mathbf{X}_{\left.\mathbf{S R S}, \mathbf{Y}_{\mathbf{S R S}}\right)}\right. \\
& +B_{F, G}(m),
\end{aligned}
$$

where

$$
\begin{aligned}
B_{F, G}(m)= & \sum_{i=1}^{m} \int_{0}^{1} \frac{[-\ln (1-u)]^{i-1}}{(i-1) !} \\
& \times \ln \left(\frac{[-\ln (1-u)]^{i-1}}{\left[-\ln \bar{G}\left(F^{-1}(u)\right)\right]^{i-1}}\right) d u \\
= & \sum_{i=1}^{m}(i-1) \psi(i) \\
& -\sum_{i=1}^{m}(i-1) \int_{0}^{\infty} \frac{z^{i-1} e^{-z}}{(i-1) !} \\
& \times \ln \left(-\ln \left(\bar{G}\left[F^{-1}\left(1-e^{-z}\right)\right]\right)\right) d z \\
= & \sum_{i=1}^{m}(i-1) \\
& \times\left[\psi(i)-E\left[\ln \left(-\ln \left(\bar{G}\left[F^{-1}\left(1-e^{-z}\right)\right]\right)\right)\right]\right],
\end{aligned}
$$

where $Z \sim \operatorname{Gamma}(i, 1)$. Note that $B_{F, G}(m)$ is dependent on the parent distributions of $X$ and $Y$ samples. Here again $K\left(\mathbf{X}_{\mathbf{S R S}}, \mathbf{Y}_{\mathbf{S R S}}\right) \leq K\left(\mathbf{U}_{\mathbf{R}}, \mathbf{V}_{\mathbf{R}}\right)$, if $B_{F, G}(m) \geq 0$.

Example 4.1. Suppose $X$ and $Y$ have exponential distribution with parameters $\theta_{1}$ and $\theta_{2}$ and cdfs $F(x)=$ $1-\exp \left(-\theta_{1} x\right)$ and $G(y)=1-\exp \left(-\theta_{2} y\right)$, respectively. We can find

$$
f\left(G^{-1}\left(1-e^{-w}\right)\right)=\theta_{1} \exp \left(-\frac{\theta_{1}}{\theta_{2}} w\right),
$$

and

$$
\begin{aligned}
A_{f, G}(m)= & \frac{-a \theta_{2}}{1+a}\left\{\frac{(m+2)(m-1)}{2}[\gamma+\ln (a+1)]\right. \\
& \left.+\sum_{i=1}^{m} \ln ((i-1) !)\right\} \leq 0
\end{aligned}
$$

where $a=\frac{\theta_{1}}{\theta_{2}} \in(0, \infty)$. Since $A_{f, G}(m) \leq 0$, we have $K\left(\mathbf{X}_{\mathbf{S R S}}, \mathbf{Y}_{\mathbf{S R S}}\right) \leq K\left(\mathbf{X}_{\mathbf{S R S}}, \mathbf{V}_{\mathbf{R}}\right)$. Also, we immediately find that

$$
\begin{aligned}
B_{F, G}(m)= & \sum_{i=1}^{m}(i-1) \psi(i) \\
& -\sum_{i=1}^{m}(i-1) \int_{0}^{\infty} \frac{z^{i-1} e^{-z}}{(i-1) !} \ln \left(\frac{\theta_{2}}{\theta_{1}} z\right) d z \\
= & \frac{(m+2)(m-1)}{2} \ln a \geq 0, \quad \text { for all } a \geq 1 .
\end{aligned}
$$

So, for the exponential distribution, $K\left(\mathbf{X}_{\mathbf{S R S}}, \mathbf{Y}_{\mathbf{S R S}}\right) \leq$ $K\left(\mathbf{U}_{\mathbf{R}}, \mathbf{V}_{\mathbf{R}}\right)$ for all $a=\frac{\theta_{1}}{\theta_{2}} \in[1, \infty)$.

\section{Conclusion}

In this paper, we consider the uncertainty and information content of RRSS data using the Shannon entropy, Rényi entropy and KL information. We show that the difference between the Shannon entropy of RRSS and SRS data is depends on the parent distribution $F$. In the sequel, we compare the Shannon entropy of RRSS data with SRS in the uniform, exponential, Weibull, Pareto, and gamma distributions. We show that if $X$ has an uniform distribution on $(0,1)$, then the record ranked set sampling provides the amount of the Rényi entropy less than simple random sampling. We also consider when $X$ has a standard exponential distribution, then the Rényi entropy of RRSS data could be bigger than the Rényi entropy of SRS data for $\lambda>1$. Also, we obtain upper bounds of Shannon and Rényi entropies for RRSS data. Finally, we show that the KL information between the distribution of $\mathbf{X}_{\mathbf{S R S}}$ and distribution of $\mathbf{U}_{\mathbf{R}}$ is distribution -free and increases as the sample size increases.

\section{Acknowledgments}

The authors are thankful to the editor and referees for helpful comments and suggestions. 


\section{Referências}

Abbasnejad, M. and Arghami, N. R. (2011). Rényi entropy properties of records. Journal of Statistical Planning and Inference, 141(7): 2312-2320.

Ahsanullah, M. (1995). Record statistics. Nova Science Publishers, New York.

Arnold, B.C., Balakrishnan, N. and Nagaraja, H.N. (1998). Records. Wiley, New York.

Baratpour, S., Ahmadi, J. and Arghami, N.R. (2007). Entropy properties of record statistics. Statisticlal Papers, 48: 197-231.

Bardlow, E. T. and Park, Y. (2007). Bayesian estimation of bid sequences in internet auction using a generalized record-breaking model. Marketing Science, 26: 218-229.

Benestad, R. E. (2003). How often can we expect a record event? Climate Research, 25: 3-13.

Chen, Z.H., Bai, Z.D. and Sinha, B.K. (2004). Ranked Set Sampling: Theory and Applications. Springer, New York.

Cover, T. M. and Thomas, J. A. (1991). Elements of information theory. John Wiley, New York.

Jafari Jozani, M. and Ahmadi, J. (2014). On uncertainty and information properties of ranked set samples. Information Sciences, 264: 291-301.

Harte, J.(2011). Maximum entropy and ecology: A theory of abundance, distribution, and energetics. Oxford University Press.

Krug, J. and Jain, K. (2005) Breaking records in the evolutionary race. Physica $A, 53: 1-9$.

Kuper, G. H. and Sterken, E. (2003). Endurance in speed skating: The development of world records. European Journal of Operational Research, 148: 293-301.

Lenzi, E.K., Mendes,R.S. and da Silva.L.R. (2000). Statistical mechanics based on Rényi entropy. Physica A, 280: 337-345.

Madadi, M. and Tata, M. (2011). Shannon information in record data. Metrika, 74: 11-31.

McIntyre, G. (1952). A method for unbiased selective sampling using ranked set sampling. Australian Journal of Agricultural Research, 3: 385-390.

Rao, M., Chen. Y., Vemuri, B. C. and Wang, F. (2004). Cumulative residual entropy: a new measure of information. IEEE Trans. Inform. Theor, 50(6): 1220-1228.
Rényi, A.(1961). On measures of entropy and information. Proceedings of Fourth Berkeley Symposium on Mathematics. Statistics and Probability 1960, vol. I. University of California Press: 547-561.

Salehi, M. and Ahmadi, J.(2014). Record ranked set sampling scheme. Metron, 72: 351-365.

Samaniego, F. J. and Whitaker, L. R.(1986). On estimating population characteristics from record-breaking observations, I. Parametric results. Naval Research Logistics Quarterly, 33: 531-543.

Shannon, C. (1948). A mathematical theory of communication. Bell System Technical Journal, 27: 379-432.

Song, K.(2001). Rényi information, loglikelihood and an intrinsic distribution measure. Journal of Statistical Planning and Inference, 93: 51-69.

Tahmasebi, S and Jafari, A.A.(2014). Information measures of ranked set samples in Farlie-GumbelMorgenstern family. Journal of Data Science, 12: 755774.

Ullah, A.(1988). Entropy, divergence and distance measures with econometric applications. Journal of Statistical Planning and Inference, 49: 137-162.

Zahedi, H. and Shakil, M. (2006). Properties of entropies of record values in reliability and life testing context. Communication in Statistics -Theory and Methods, 35: 997-1010. 